\title{
Elimination and Prevention of Microbial Colonization of Central Venous Catheters Using Antibiotic Lock Technique and Non- Leachable Form of Catheter Surface Incorporated Antibiotic by Gamma Radiation
}

\author{
Hala A. Farrag ${ }^{1}$, Alaa El-Dien M.S. Hosny ${ }^{2}$, Amr El Hag Ali ${ }^{3}$, \\ Soheir A.A. Hagras ${ }^{4}$ \\ ${ }^{1}$ Professor of Microbiology, Drug Radiation Research Dept., National Center for Radiation Research and \\ Technology, Cairo, Egypt. \\ ${ }^{2}$ Professor of Microbiology, Microbiology \& Immunology Dept.., Faculty of Pharmacy, Cairo University, Cairo, \\ Egypt. \\ ${ }^{3}$ Professor of Polymer Chemistry, Polymer Chemistry Dept. National Center for Radiation Research and \\ Technology, Cairo, Egypt. \\ ${ }^{4}$ Assistant Lecturer, Drug Radiation Research Dept., National Center for Radiation Research and Technology,
} Cairo, Egypt.

\begin{abstract}
Aim: To test the efficacy of antibiotic lock technique (ALT) and catheter material modification for the eradication and prevention of biofilm formation on central venous catheters (CVCs). Methods: Four resistant clinical isolates, namely Pseudomonas aeruginosa, Acinetobacter baumannii, Klebsiella pneumoniae and Candida tropicalis were used to study the effectiveness of ALT using levofloxacin (for bacteria) or fluconazole (for candida) to eliminate microbial biofilms. Moreover, with the aid of gamma radiation, gentamicin was covalently attached to the polyurethane catheter and the modified polymeric material was tested for their antimicrobial and anti-adherence properties. Results: The highest and lowest concentrations of levofloxacin tested (1024 and 64 $\mathrm{mg} / \mathrm{L})$ eliminated the biofilms of Pseudomonas aeruginosa and Acinetobacter baumannii after 3 and 10 days, respectively and after 1 and 7 days in case of Klebsiella pneumoniae, whereas Candida tropicalis biofilms were eliminated by fluconazole after 3 and 10 days. The modified polymeric material acquired antibacterial and antiadherence properties. Conclusion: The results suggest that ALT and catheter modification could be effective for eradicating and preventing biofilm formation on CVCs.
\end{abstract}

Key words: Biofilm; Antibiotic lock technique; Central venous catheter; Glycidyl methacrylate, polymer modification.

\section{Introduction}

The central venous catheter (CVC) is an important tool in modern medical practices. It provides secure access to the central circulation for infusion therapy, nutritional support, hemodynamic monitoring, plasmapheresis and hemodialysis. The use of central venous catheters (CVCs) is associated with increased risk of catheter-related blood stream infections (CRBSIs). They have a higher infection risk than other indwelling vascular access lines (Patil et al 2011). The infective agents, causing such infections, colonize the catheter surface forming biofilm and resulting in increased rates of morbidity and mortality, and prolonged hospitalization periods (Rodney 2011;Cherifi et al 2013 and Kostakioti et al 2013). Aside from septic complications, failure to prevent or eradicate microbial contaminants from catheter surfaces may result in phlebitis, skin infections, and/or catheter loss and removal (Shirtliff et al 2009). However, catheter removal may not be a viable option if thrombocytopenia, coagulopathies, a lack of alternative venous access, or critical illness is a confounding issue. In addition, the clinical need for these catheters in some patients, including parenteral nutrition and intravenous drips, further complicates the decision of catheter removal. Therefore, alternative strategies are needed to prevent contamination or permit salvage of catheters in these clinical situations (Peters et al, 2013).One current approach is the use of antimicrobial catheter lock solutions, which are formulated to sterilize the catheter lumen or prevent biofilm growth and subsequent septic infection (Toltzis 2006). Several studies recommended ALT to prevent catheter-related infections (Casey and Elliott 2010 and Kulawik 2010).

Polyurethane is widely used in vascular catheters, given its favorable biocompatibility and excellent mechanical properties (Yuan et al 2004). However, it is slightly hydrophobic, a characteristic that facilitates bacterial adherence and biofilm formation (Egboh et al 1984). Modification of the biomaterial surface was regarded the most promising preventive strategy for biofilms. The covalent immobilization of the antimicrobial agent offers many privileges, including long term stability and protection against bacterial colonization, lowered toxicity and 
decreased risk of emergence of antimicrobial resistant strains (Makal et al 2006; Humblot et al 2009 and Xu et al 2009). The aim of this study was to evaluate the efficacy of ALT and catheter material modification to eradicate and prevent microbial biofilms.

\section{Equipments:}

\section{Materials and Methods}

Incubatory Shaker (Lab-Line Instruments Inc., Melrose, USA),

$\mathrm{pH}$ Meter (Jenway Ltd. UK), Vortex (Gemmy Industrial Corp., Taiwan), VCX-400 Sonicator (Sonics \& Materials Inc. Danbury. CT, USA), Gamma Radiation Cell $\left({ }^{60} \mathrm{Co}-\right.$ Atomic Energy of Russian'), IR Spectroscopy (FTIR Jasco 9200 Japan) and Scanning Electron Microscopy (JEOL JSM-5400, Japan).

\section{Culture media:}

Nutrient agar, MacConkey agar, Sabouraud dextrose agar and trypticsoy broth. All the media were the products of Oxoid Ltd. (England). The media were autoclaved at $121^{\circ} \mathrm{C}$ for 20 minutes. Heat labile components were separately sterilized by filtration and aseptically added to the medium.

\section{Microbial Strains:}

Three clinical bacterial isolates, namely Acinetobacterbaumannii (A. baumannii), Pseudomonas aeruginosa (P. aeruginosa) and Klebsiella pneumoniae (K. pneumoniae) in addition to a Candida tropicalis (C. tropicalis) isolate. The test strains were selected among 117 isolates previously isolated from 83 CVCs collected during 2006 from ICU patients. They were selected based on their antibiotic resistance and slime productivity.

\section{Antimicrobial Agents:}

Levofloxacin and fluconazole were used as antibiotic lock solutions for bacteria and yeast, respectively. For modification of catheter polymeric material, gentamicin was used. These antimicrobial agents were obtained from the National Organization for Drug Control and Research (NODCAR), Egypt.

5. Catheters: Polyurethane central venous (CVPU) cathetersare a product of (AmeCath., Egypt).

6. Chemicals:

6.1 Glycidyl methacrylate (GMA); A transparent colorless liquid, contains carbon-carbon double bond and an epoxy group which make free radical reaction and ionic reaction occur. It was the product of Fluka, Biochemika, Switzerland.

6.2 Solvents used: acetone, methanol, and ethyl acetate, all analytical grade reagents (Romil ltd., GB).

\section{Antibiotic- Lock Experiments:}

\subsection{Biofilm formation on catheter surface}

Mature biofilms on CVPU fragments were formed using the method of Golomb and Shpigelman (1991). Briefly, sterile segments were cut and suspended at $30^{\circ} \mathrm{C} / 5$ days in microbial suspensions $\left(10^{7} \mathrm{CFUs} / \mathrm{ml}\right)$ using tryptic soy broth supplemented with $0.25 \%$ sucrose in incubatory shaker, at $100 \mathrm{rpm}$.

\subsection{Model of antimicrobial lock technique}

The method of Lee et al (2006 and 2007) was applied. Briefly, segments with biofilms were washed three times with phosphate buffered saline (PBS), placed in fresh $10 \mathrm{ml}$ tryptic soy broth supplemented with $0.25 \%$ sucrose, containing different concentrations $(64-1024 \mathrm{mg} / \mathrm{L})$ of Levofloxacin for bacteria or fluconazole for Candida tropicalis. At suitable time intervals of 1, 3, 5, 7 or 10 days, three segments were picked up from each tube, washed three times with PBS, vortexed and sonicated separately in $3 \mathrm{ml}$ fresh PBS for 2 minutes using VCX-400 sonicator (Sonics \& Materials Inc. Danbury. CT, USA) with a pulse rate of 3.5 seconds to separate and re-suspend the adherent cells, as confirmed by scanning electron microscopy (SEM). Then the sonicated microbial suspension was vortexed and serially diluted, and the viable count was determined using agar plate technique. The experiments were done in triplicate and the effect of concentration and contact time was statistically analyzed using F-test.

\subsection{Scanning Electron Microscopy (SEM) Studies}

Scanning electron microscopy (SEM) studies, using the method of Storti et al (2005), were carried out on $P$. aeruginosa or $C$. tropicalis for the lock concentrations of 64,256 or $1024 \mathrm{mg} / \mathrm{L}$ and compared to the antibiotic free PBS.

\section{Modification of Catheter Material by Gentamicin}

\subsection{Graft copolymerization of GMA on to CVPU (GMA-g-CVPU) (Fig 1a).}

A method similar to that used by Egboh et al (1984) was performed to carry out radiation-induced graft copolymerization of glycidyl methacrylate (GMA) onto segments of polyurethane (PU) catheters. Graft copolymerization reaction was carried out in different solvents, including acetone, ethyl acetate or methanol. In brief, polyurethane segments were washed with acetone, dried at $50^{\circ} \mathrm{C}$ in a vacuum oven, weighed, and then 
immersed in GMA solution in glass ampoules. The reactant solutions were de aerated by nitrogen atmosphere to remove air from grafting solution. The sealed glass ampoules were then subjected to ${ }^{60} \mathrm{Co}-\gamma$ rays. The grafted films were removed and washed thoroughly with methanol/DMF to extract the residual monomer and homopolymer, which may be occluded in the segments. The films were then dried in a vacuum oven at $40-50^{\circ} \mathrm{C}$ for $24 \mathrm{~h}$ and weighed. The degree of grafting (DG) was determined as it follows;

$$
\mathrm{DG}(\%)=\left[\mathrm{W}_{\mathrm{g}}-\mathrm{W}_{\mathrm{o}} / \mathrm{W}_{\mathrm{o}}\right] \times 100
$$

Where $\mathrm{W}_{\mathrm{o}}$ and $\mathrm{W}_{\mathrm{g}}$ are the weight of the ungrafted and grafted segments, respectively.

\subsection{Chemical immobilization of gentamicin on (GMA-g-CVPU) (Fig 1b).}

Covalent bonding of gentamicin, to the grafted segments, was performed by refluxing with super saturated alcoholic gentamicin solution, at $70^{\circ} \mathrm{C} / 4 \mathrm{hrs}$. in presence of few drops of dimethyl formamide, to enhance solubility of gentamicin. The treated catheter segments were then washed and dried. The conversion to amine structure was determined by the disappearance of the epoxy groups to secondary amine groups by FTIR spectra (Fig 6).

\subsection{Fourier Transform Infrared (FTIR) Spectroscopy}

A commercial FTIR (Jasco 9200 Japan) spectroscopy, at scanning range of 4000 to $200 \mathrm{~cm}^{-1}$ wave number, was employed to investigate the chemical characteristics of virgin PU, graft copolymer (CVPU-g-GMA) and gentamicin-modified grafted films (CVPU-g-GMA-Gentamicin).

\subsection{Antimicrobial Effectiveness}

\subsubsection{Test for antimicrobial activity of graft copolymer and modified copolymer:}

The graft copolymer (CVPU-g-GMA), gentamicin-modified copolymer (CVPU-g-GMA-Gentamicin) and polyurethane (CVPU), as a control, were tested for their antimicrobial activity by placing strips of these polymers on agar plates inoculated with $P$. aeruginosa, A. baumannii or $K$. pneumoniae. After incubation, plates were examined for any inhibition zones around the strips or their contact inhibition zones.

\subsubsection{Test for the anti-adherence capacity of gentamicin- modified copolymers:}

The anti-adherence capacity of graft copolymer (CVPU-g-GMA), gentamicin-modified copolymers (CVPU-g-GMA-Gentamicin) and polyurethane (PU), as a control, was tested against highly slime producer bacterial strains used above in the lock experiment. Briefly; segments were placed separately, in microbial suspension $\left(10^{8} \mathrm{CFUs} / \mathrm{ml}\right)$ of these organisms, incubated at $37^{\circ} \mathrm{C}$ and examined for biofilm formation during 30 days, at time interval of five days. Where; three segments from each suspension were washed with PBS, sonicated to re-suspend any adherent bacterial cells (Sherertz et al 1990). After sonication, aliquots were well mixed using high speed vortex, 10 fold serially diluted and $0.1 \mathrm{ml}$ from each dilution was inoculated on the surface of nutrient agar plates. The number of colonies was counted after $24-48$ hours incubation at $37^{0} \mathrm{C}$. Results of test and control were confirmed using the scanning electron microscopy as described before.

\section{Effect of levofloxacin as a lock solution on bacterial biofilms: \\ III. Result}

The results of levofloxacin on bacterial biofilms of initial counts of $4.5 \times 10^{7}, 1.7 \times 10^{7}$ and $1.3 \times 10^{8}$ CFUs/segment for $P$. aeruginosa, A. baumannii and K. pneumoniae, respectively, showed that the count was decreasing by time, where a concentration of $1024 \mathrm{mg} / \mathrm{L}$ eliminated their biofilms within 3 days and a concentration of $64 \mathrm{mg} / \mathrm{L}$ within 10 days. Conversely, the elimination time was earlier for K. pneumoniae (7 days), and various elimination times were recorded for other concentrations tested (128-512 mg/L) (Fig 2a, b, c). The results were confirmed for $P$. aeruginosa by SEM (Fig 3).

\section{Effect of fluconazole as antifungal lock solution:}

As for bacteria, an initial biofilm count of $5.3 \times 10^{6} \mathrm{CFUs} /$ segment for $C$. tropicalis was decreasing by time (Fig. 2d) and complete elimination was recorded and confirmed by SEM (Fig. 4) within 3 and 10 days for concentrations of 1024 and $64 \mathrm{mg} / \mathrm{L}$, respectively.

Effects of radiation dose, solvent and monomer concentration (GMA) on the grafting yield:

Among radiation doses of 10,20 and $30 \mathrm{kGy}$, different solvents (acetone, ethyl acetate and methanol), as well as GMA concentrations of 20 and $30 \%$ used ,the results showed that the degree of grafting increased with the increase in monomer concentration (up to $30 \%$ ) and radiation dose up to $30 \mathrm{kGy}$ (Fig. 5). For different solvents, the grafting yield was $<20 \%$ for acetone, $<10 \%$ for ethyl acetate, while the best yield (> 50\%) was recorded for methanol. 


\section{Modification of graft copolymer by gentamicin:}

Figure (6) shows the FTIR spectrum of CVPU-g-GMA copolymer in comparison with that of the original CVPU. CVPU spectrum (Fig 6 a) shows its characteristic peaks at 3294.8 and $1536.1 \mathrm{~cm}^{-1}$ assigned to NH absorption, $2880-2890 \mathrm{~cm}^{-1}$ attributed to $\mathrm{C}-\mathrm{H}$ symmetric and asymmetric stretching absorption and $1704.0 \mathrm{~cm}^{-}$ ${ }^{1}$ attributed to the urethane - NHCOO- carbonyl absorption. Upon grafting of GMA (Fig 6 b) a new absorption band appeared at $1724 \mathrm{~cm}^{-1}$ belonging to the $\mathrm{C}=\mathrm{O}$ ester and at 1193 and $1159 \mathrm{~cm}^{-1}$ which belonged to the epoxy group of the grafted GMA (Xifei 2001). This confirmed the occurrence of the grafting process by showing the absorption peaks of grafted function groups together with that of polymer substrate. Figure (6 c) shows in addition to the absence of the absorption band belong to the epoxy ring, some broadness in the absorption peaks at 33003500 due to the appearance of $-\mathrm{OH}$ as result of epoxy ring opening. Such information shows that the epoxy group in the side chain of CVPU-g-GMA has reacted with the functional (NH2) group of gentamicin. The above observations verified the successful covalent attachment of gentamicin to the CVPU. This was also confirmed microbiologically, where only gentamicin modified polymer showed contact inhibition zones against the three test bacterial strains, on inoculated agar plates, as well as prevention of their adherence to the modified polymer surface when suspended in their broth culture, as confirmed by scanning electron microscopy (Fig 7).

\section{Discussion}

Biofilm formation is the main problem in the pathogenesis of catheter-related infections. These biofilms cannot be penetrated by antibodies or phagocytes and are 1500 times less susceptible to antibiotics than are their planktonic counterparts. This led to targeted therapy aimed at eliminating the biofilms while salvaging the catheter (Costerton et al 1999 and Donlan, 2001). The concepts behind antibiotic lock technique (ALT) are to prolong the life of the catheter and reduce morbidity and costs of managing catheter-related infections. In addition, it may spare patients from certain toxic effects of systemic antibiotic administration, and the risk of bacterial resistance may be reduced (Capdevila et al 1996 and Oppenheim, 2000). ALT is especially beneficial for patients with underlying comorbidities complicating catheter removal or patients who are catheter dependent. It is also indicated for central line associated bloodstream infections involving long-term catheters with no signs of exit site or tunnel infection with the goal of catheter salvage (Estes et al 2013 and Peters et al 2013). In vitro study for prevention and/or elimination of bacterial and fungal biofilms on central venous catheters, especially long term ones, was performed. As the lock solutions are trapped inside the catheter without any systemic effect, so the use of as high as 23-512 folds of the MIC of the lock antibiotics was expected to be a highly effective and safe technique. The elimination of biofilm was concentration dependent; levofloxacin eliminated $P$. aeruginosa and $A$. baumannii biofilms within 3 days and up to 10 days at concentrations of about $1 \mathrm{mg} / \mathrm{ml}$ and $0.06 \mathrm{mg} / \mathrm{ml}$, respectively. However, the elimination time was shorter for $K$. pneumoniae ( $24 \mathrm{hrs}$ and 7 days) at the same concentrations. Similar elimination rate was reported by Lee et al (2007) for $P$. aeruginosa using ciprofloxacin as a lock solution.

Elimination of $C$. tropicalis biofilms, by fluconazole, showed similar response to that of $P$. aeruginosa and $A$. baumannii (within 3 and 10 days at similar concentrations). Similar observations were recorded but at longer duration ( $1 \mathrm{mg} / \mathrm{ml}$ at 7 days) by Ko et al (2010) and higher concentration ( $2 \mathrm{mg} / \mathrm{ml} \mathrm{at} 7$ days) by Andris $e t$ al (1998).

As shown by the SEM, lock solutions (either levofloxacin or fluconazole), eliminated both microbial cells within biofilm and the expolymeric material around them. It is an advantage for the lock technique to target both biofilm components (matrix and microbial population), so it not only eliminates biofilm but also prevents catheter re-colonization. Similar observation was reported by Totéa et al (2009).

Although polyurethane has excellent mechanical and anti-thrombogenic properties, nevertheless its hydrophobicity facilitates adherence and biofilm formation. Different methods were developed for synthesis of antibacterial surfaces. Covalent bonding of active antimicrobial agents to the substrate surface is a useful approach for the development of non-leaching or contact biocide surface. The antimicrobial action results from the contact of the microorganisms with the surface without release. Cationic antimicrobial agents play a key role in the development of permanent and non-leaching contact biocidal surfaces where they affect the integrity of the negatively charged bacterial cells, causing release of the intracellular components (Biering-Sorensen, 2002; Lee $e t$ al 2004; Makal et al 2006; Murata et al 2007 and Xu et al 2009). Gamma radiation is often used to sterilize 
polymeric medical devices at doses exceeding $15 \mathrm{kGy}$. Commercial polyurethanes show good resistance to highenergy radiation, withstanding a single dose of up to $100 \mathrm{kGy}$ (Gorna and Gogolewski 2003).

In the present study, the grafting yield of GMA onto CVPU increased with increasing GMA concentration and the radiation dose. The optimum conditions were the use of a radiation dose of $30 \mathrm{kGy}, 30 \% \mathrm{GMA}$, and methanol as a solvent. The increase in grafting yield may be attributed to the increase in free radicals concentration that arises from the increase in the monomer concentration (Charlesby, 1960) and hence increase in the epoxy groups' content on polyurethane that act as surface linkers for the nucleophilic amino group of gentamicin. Other nucleophilic groups, as linkers, including $\mathrm{NH} 2, \mathrm{OH}, \mathrm{SH}$ were reported by others (Eckert et al 2000 and Nishiyama et al 2002). On the other hand, methanol facilitates the accessibility of GMA to the polymer active site; similar results were recorded by Inderjeet et al (1999) and El-Hag Ali et al (2008). In spite of being effective, the maximum radiation dose used was $30 \mathrm{kGy}$, because at a higher dose (50 kGy) the physical properties of GMA monomer were affected and yielded a powdery form. Similar observation was reported by Jansen and Ellinghorst (1979), who observed gelation of GMA.

Being a heat stable antibiotic (up to $100 \mathrm{C}$ ) (Wang et al 2004), gentamicin was covalently immobilized into graft copolymer by refluxing at $70 \mathrm{C}^{\circ} / 4 \mathrm{hrs}$, as confirmed by FTIR. The immobilized antibiotic retained its antibacterial activity as confirmed by contact inhibition zone. The persistence of the antimicrobial activity of immobilized gentamicin may be explained by its flexibility due to the presence of a linker spacer (Liu and Ming, 2008 and Humblot et al 2009). In addition; gentamicin was reported to have a surface action on bacterial cell envelope (Kadurugamuwa et al 1993 a, b). In conclusion, our study suggests that both ALT and catheter polymer modification could be effective in eradicating and preventing microbial biofilms on CVCs, hence preventing catheter related infections ; however, further studies are required to verify their effectiveness in vivo.

\section{Acknowledgment}

We thank Mr. Dante Frederick, English language educator, IPA, KSA, for reviewing the paper for the specificity of English language.

\section{References:}

[1]. Andris DA, Krzywda EA, Edmiston CE, Krepel CJ and Gohr CM (1998) Elimination of intraluminal colonization by antibiotic lock in silicone vascular catheters. Nutrition 14(5), 427-432.

[2]. Biering-Sorensen F. (2002). "Urinary tract infection in individuals with spinal cord lesion. Curr. Opin. Urol. 12, 45-49.

[3]. Capdevila JA, Gavalda J and Pahissa A (1996) Antibiotic-lock technique: usefulness and controversies. Antimicrob. Infect. Dis. News 15, 9-16.

[4]. Casey AL and Elliott TS (2010) Prevention of central venous catheter-related infection: update. Br. J. Nurs. 19:78-87.

[5]. Charlesby A (1960) Atomic Radiation and Polymers. Pergamon Press, Oxford.

[6]. Cherifi S, Mascart G, Dediste A, Hallin M, Gerard M, Lamber ML and Byl B (2013) Variations in catheter-related bloodstream infections rates based on local practices. Antimicrobial resistance and infection control 2(10).

[7]. Costerton JW, Stewart PS and Greenberg EP (1999) Bacterial biofilms: A common cause of persistent infections. Science 284, 1318-1322.

[8]. Del Pozo JL (2009) Role of antibiotic lock therapy for the treatment of catheter-related blood stream infections. Int. J. Artif. Organs 32:678-688.

[9]. Donlan RM (2001). "Biofilm formation: A clinically relevant microbiologic process. Clin. Infect. Dis. 33, 1387-1392.

[10]. Eckert AW, Grobe D and Rothe U (2000) Surface-modification of polystyrene-microtitre plates via grafting of glycidylmethacrylate and coating of poly-glycidyl methacrylate. Biomaterials 21, 441-447.

[11]. Egboh SH, George MH and Barrie JA (1984) The gamma radiation induced grafting of unsaturated segmented polyurethane with $\mathrm{N}$-vinyl pyrrolidone. Polymer 25, 1157-1160.

[12]. El-Hag Ali A, Shawky HA, El-Sayed MH and Ibrahim H (2008) Radiation synthesis of functionalized polypropylene fibers and their application in the treatment of some water resources in western desert of Egypt. J. Sep. Pur. Tech. 63 (1), 69-76.

[13]. Estes R, Theusch J, Beck A, Pitrak D and Mullanea KM (2013) Activity of daptomycin with or without 25 percent ethanol compared to combinations of minocycline, EDTA, and 25 percent ethanol against methicillin-resistant Staphylococcus aureusisolates embedded in biofilm. Antimicrobial Agents and Chemotherapy. 57 (4), 1998-2000

[14]. Golomb G and Shpigelman A (1991) Prevention of bacterial colonization on polyurethane in vitro by incorporated antibacterial agent. J. Biomed. Mater. Res. 25(8), 937-952.

[15]. Gorna K and Gogolewski S (2003) The effect of gamma radiation on molecular stability and mechanical properties of biodegradable polyurethanes for medical applications. Poly. Degrad. Stab. 79, 465-474.

[16]. Humblot V, Yala JF, Thebault P, Boukerma K, He'quet A, Berjeaud JM and Pradier CM (2009) The antibacterial activity of Magainin I immobilized onto mixed thiols Self-Assembled Monolayers. Biomaterials 30, 1-10.

[17]. Inderjeet K, Sunil K, Misra BN and Ghanshyam S (1999). J. Materials Science and Engineering, 270, 137-144.

[18]. Jansen B and Ellinghost G (1979). Polym. Sci., Polym. Symp. 66, 465.

[19]. Kadurugamuwa JL, Clarke AJ and Beveridge TJ (1993b) Surface action of gentamicin on P. aeruginosa. J. Bacterio. 175(18), 57985808 . 
[20]. Kadurugamuwa JL, Lam JS and Beveridge TJ (1993a) Interaction of gentamicin with the A band and B band lipopolysaccharides of P. aeruginosaand its possible lethal effect. J. Antimicrob. Chemother. 37, 715-721.

[21]. Ko KS, Lee JK, Song JH and Peck KR (2010) In vitro evaluation of antibiotic lock technique for the treatment of C. albicans, C. glabrata, and C. tropicalisbiofilms. J Korean Med Sci; 25: 1722-1726.

[22]. Kostakioti M, Hadjifrangiskou M and Hultgren SJ (2013) Bacterial biofilms: development, dispersal, and therapeutic starategies in dawn of the postantibiotic era. Cold spring harbperspect med 3:a010306.

[23]. Kulawik D (2010) Antimicrobial lock solutions: an approach to reduce catheter-related bacteremia. Nephrol. Nurs. J. 37:553-555.

[24]. Lee JY, Ko KS, Peck KR, Oh WS and Song JH (2006) In vitro evaluation of the antibiotic lock technique (ALT) for the treatment of catheter-related infections caused by staphylococci. J AntimicrobChemother.; 57(6): 1110-1115.

[25]. Lee MY, Ko KS, Song J and Peck KR (2007) In vitro effectiveness of the antibiotic lock technique (ALT) for the treatment of catheter-related infections by Pseudomonas aeruginosa and Klebsiellapneumoniae. J. Antimicr. Chemother. 60, $782-787$.

[26]. Lee SB, Koepsel RR, Morley SW, Matyjaszewski K, Sun Y and Russell AJ (2004) Permanent, non-leaching antibacterial surfaces synthesis by atom transfer radical polymerization. Bio macromolecules 5, 877-882.

[27]. Liu H and Ming X (2008) Polymers having covalently bound antibiotic agents. WO 2008137519 A1.

[28]. Makal U, Wood L, Ohman DE and Wynne KJ (2006) Polyurethane biocidal polymeric surface modifiers. Biomaterials 27, 13161326.

[29]. Murata H, Koepsel RR, Matyjaszewski K and Russell AJ (2007) Permanent, non-leaching antibacterial surfaces-2: How high density cationic surfaces kill bacterial cells. Biomaterials 28, 4870-4879.

[30]. Nishiyama S, Goto A, Saito K, Sugita K, Tanmada M, Sugo T, Funami T, Goda Y and Fujimoto S (2002) Concentration of 17estradiol using an immune-affinity porous hollow-fiber membrane. Anal. Chem. 74, 4933-4936.

[31]. Oppenheim BA (2000) Optimal management of central venous catheter-related infections: what is the evidence?. J. Infect. 40, 2630.

[32]. Patil HV,Ramteerthkar MN, and Kulkarni RD(2011)Central venous catheter-related bloodstream infections in the intensive care unit. Indian J Crit Care Med.; 15(4): 213-223.

[33]. Peters BM, Ward RM, Rane HS, Lee SA and Noverr MC (2013) Efficacy of ethanol against Candida albicans and Staphylococcus aureuspolymicrobial biofilms. Antimicrobial Agents and Chemotherapy, 57 (1): 74-82.

[34]. Rodney MD (2011) Biofilm elimination on intravascular catheters: important considerations for the infectious disease practitioner. CID 52 (8). 1038-1045.

[35]. Sherertz RJ, Raad, ABelani, Koo LC, Rand KH, Pickett DL, Straub SA and Fauergach LL (1990) Three-year experience with sonicated vascular catheter cultures in a clinical microbiology laboratory. J. Clin. Microbiol. 28:76-82.

[36]. Shirtliff ME, Peters BM and Jabra-Rizk MA (2009) Cross-kingdom interactions: Candida albicans and bacteria. FEMS Microbiol. Lett. 299:1-8.

[37]. Storti A, Pizzolitto AC and Pizzolitto EL (2005) Detection of mixed microbial biofilms on central venous catheters removed from intensive care unit patients. Braz. J. Microb. 36, 275-280.

[38]. Toltzis P (2006) Antibiotic lock technique to reduce central venous catheter-related bacteremia. Pediatr. Infect. Dis. J. 25:449-450.

[39]. Totéa K, Vanden-Berghea D, Deschachta M, deWita K, Maesa L and Cosa P (2009) Inhibitory efficacy of various antibiotics on matrix and viable mass of Staphylococcus aureusand Pseudomonas aeruginosabiofilms. Int. J. Antimicr. Agents 33, 525-531.

[40]. Wang G, Liu SJ, Ueng SWN and Chan ER (2004) The release of cefazolin and gentamicin from biodegradable PLA/ PGA beads. Int. J. Pharmaceutics 273, 203-212.

[41]. XifeiG (2001) The research of the GMA Grafting the HDPE", Synthetic Mat Aging Appl, vol. 4, 17-19.

[42]. Xu FJ, Neoh KG and Kang ET (2009) Bioactive surfaces and biomaterials via atom transfer radical polymerization. J.PPolysci. 34, 719-761.

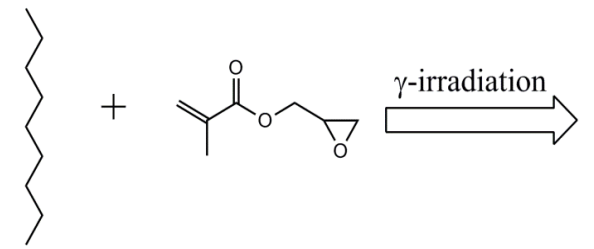

$\mathrm{PU}$

GMA

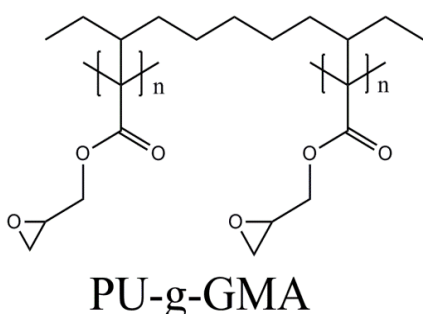

PU-g-GMA

Fig 1a. Schematic representation of the radiation induced grafting of GMA onto CVPU segments

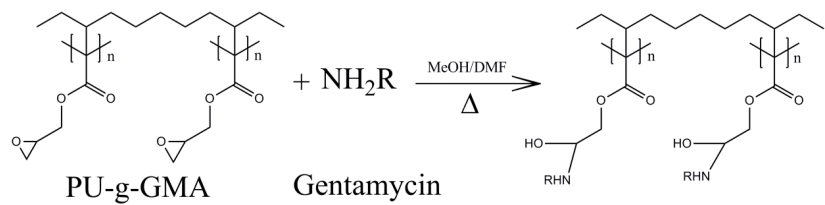

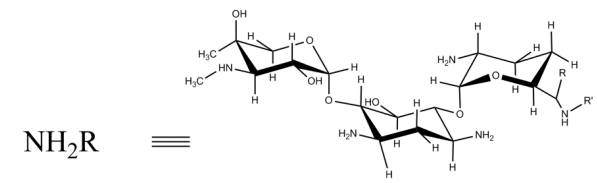

Fig 1b. Schematic representation of the chemical immobilization of gentamicin to the radiation modified CVPU catheter 


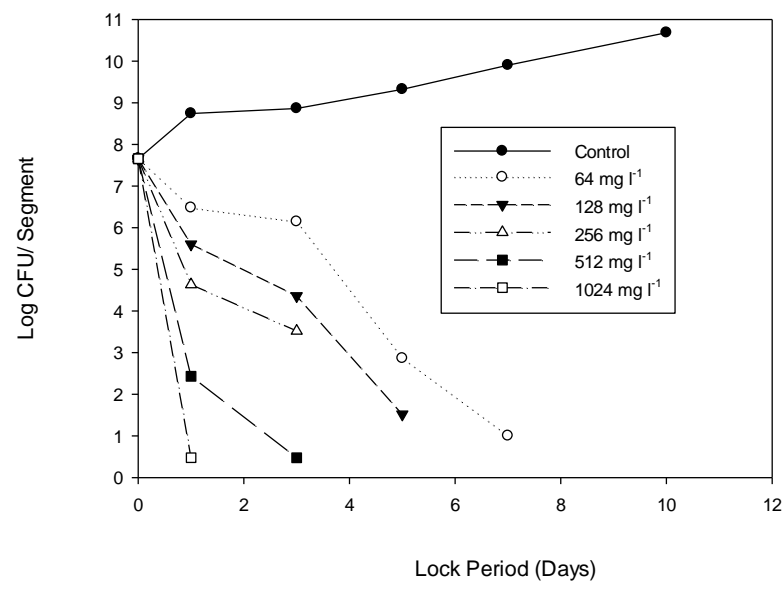

Fig 2a. Change in microbial count of $P$. aeruginosa biofilm in presence of different concentrations of levofloxacin at different lock periods.

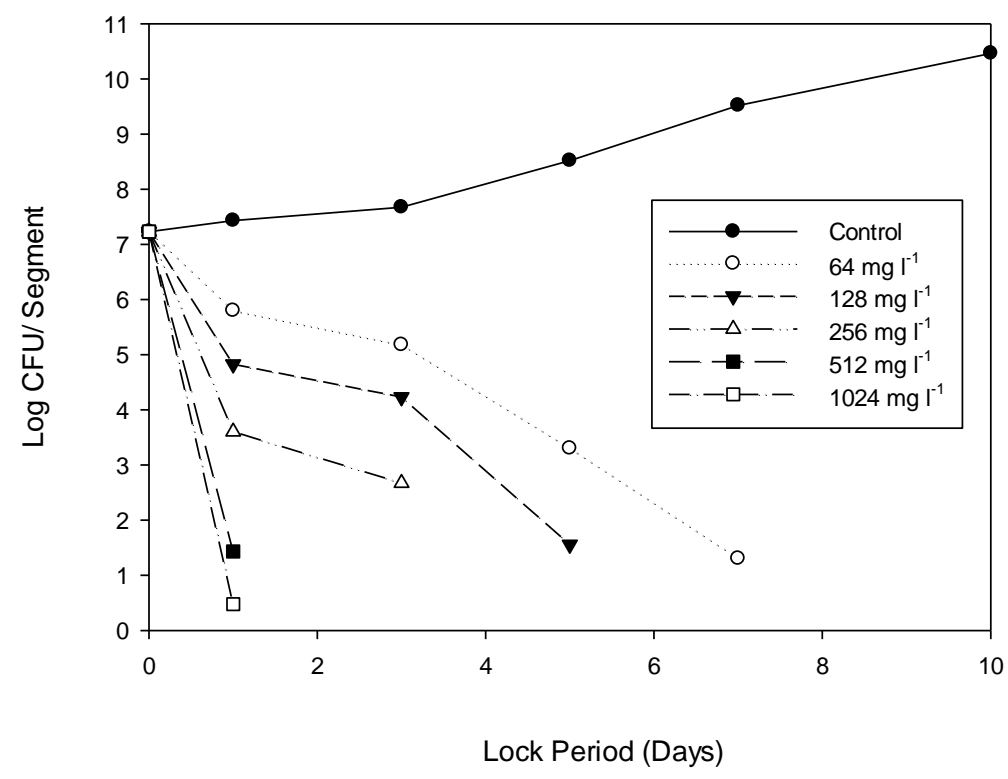

Fig 2b. Change in microbial count of $A$. baumannii biofilm in presence of different concentrations of levofloxacin at different lock periods.

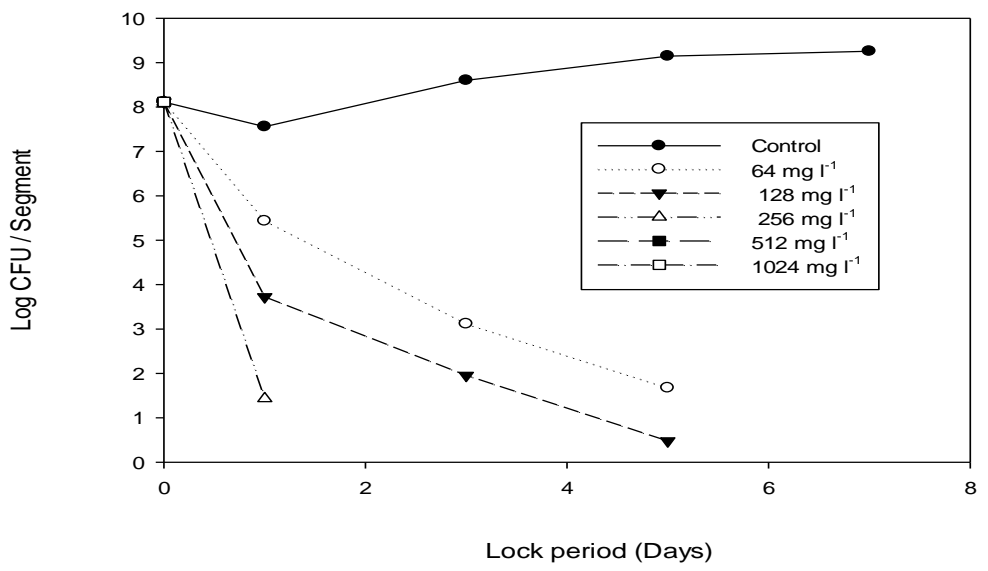

Fig 2c. Change in microbial count of $K$. pneumoniae biofilm in presence of different concentrations of levofloxacin at different lock periods. 


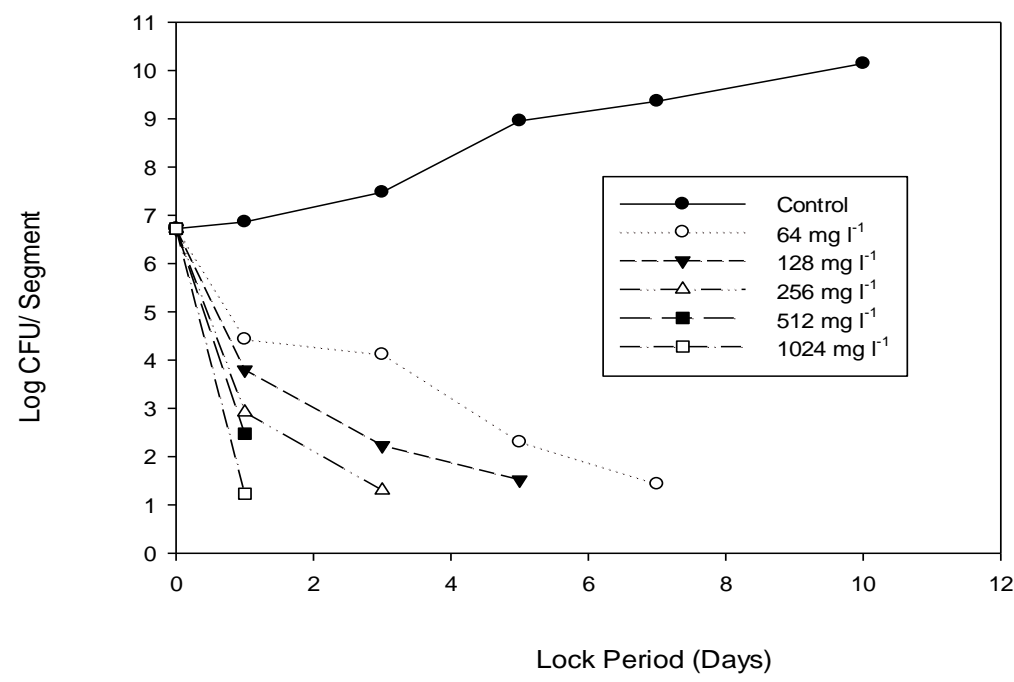

Fig 2d. Change in microbial count of $\boldsymbol{C}$. tropicalis biofilm in presence of different concentrations of fluconazole at different lock periods
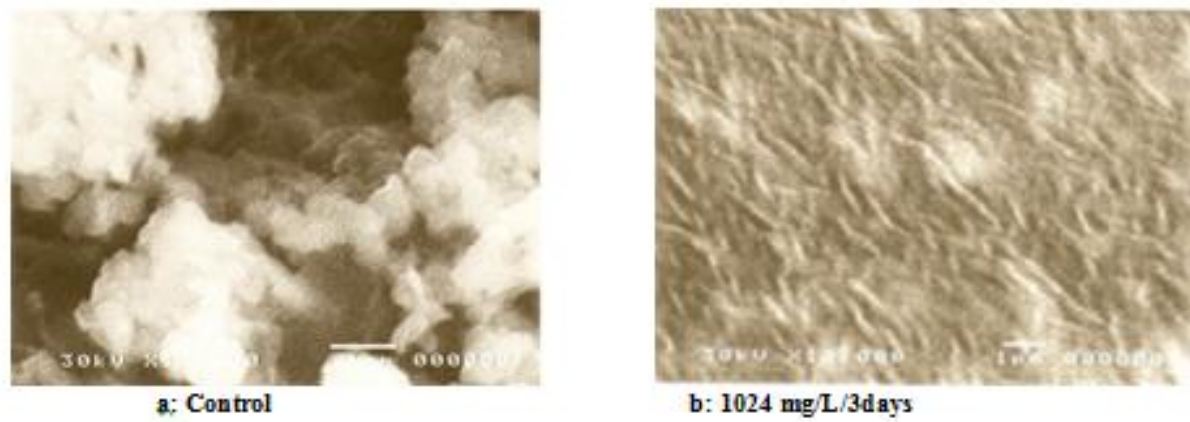

b: $1024 \mathrm{mg} / \mathrm{L} / 3 \mathrm{~d}$ ays
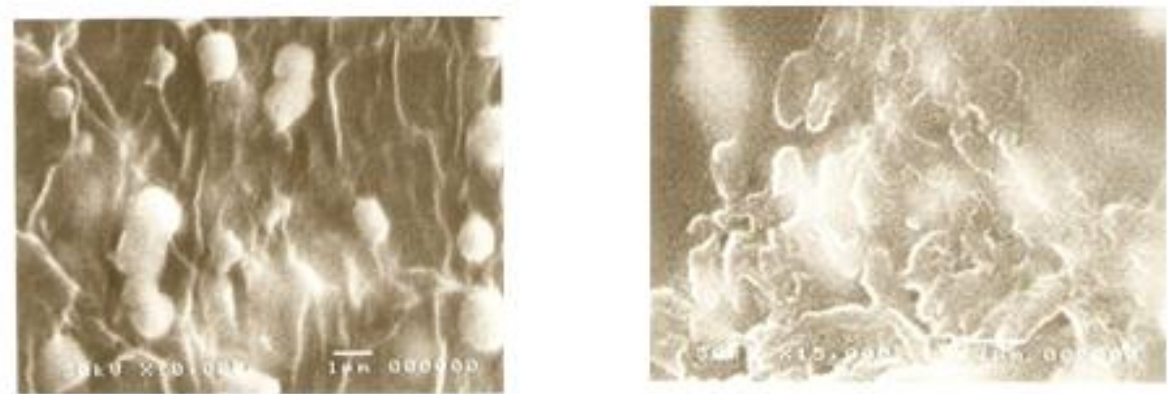

c: $256 \mathrm{mg} / \mathrm{L} / 3$ days

d: $64 \mathrm{mg} / \mathrm{L} / 3$ days

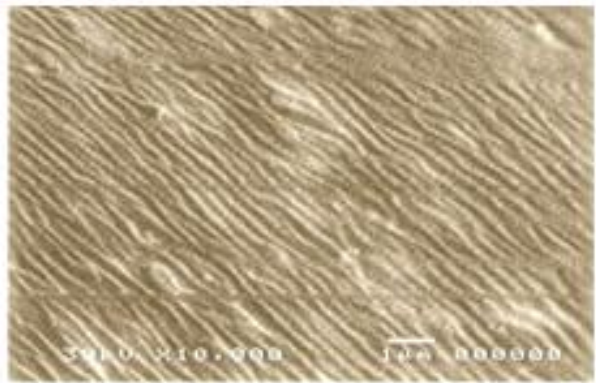

e: $64 \mathrm{mg} / \mathrm{L} / 10 \mathrm{days}$

Fig 3. Scanning electron micrographs of $P$. aeruginosa biofilm after different lock periods 

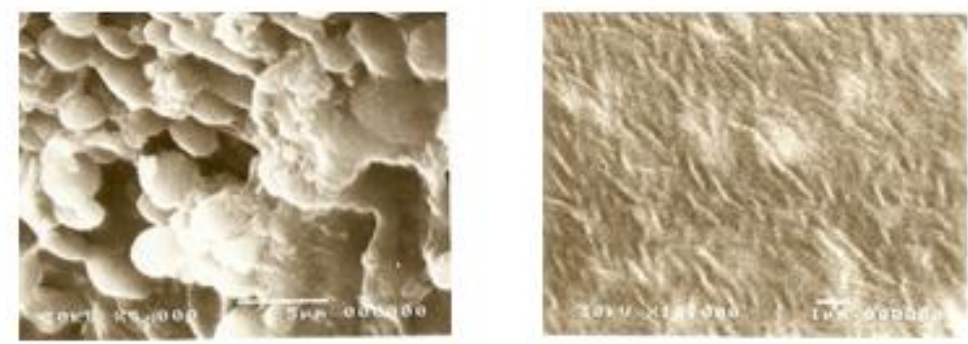

a: Control

b: $1024 \mathrm{mg} / \mathrm{L} / 3$ days

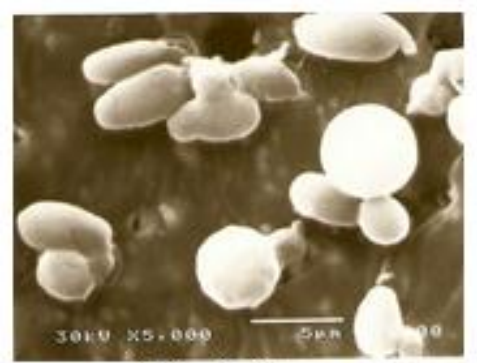

c: $256 \mathrm{mg} / \mathrm{L} / 3$ days
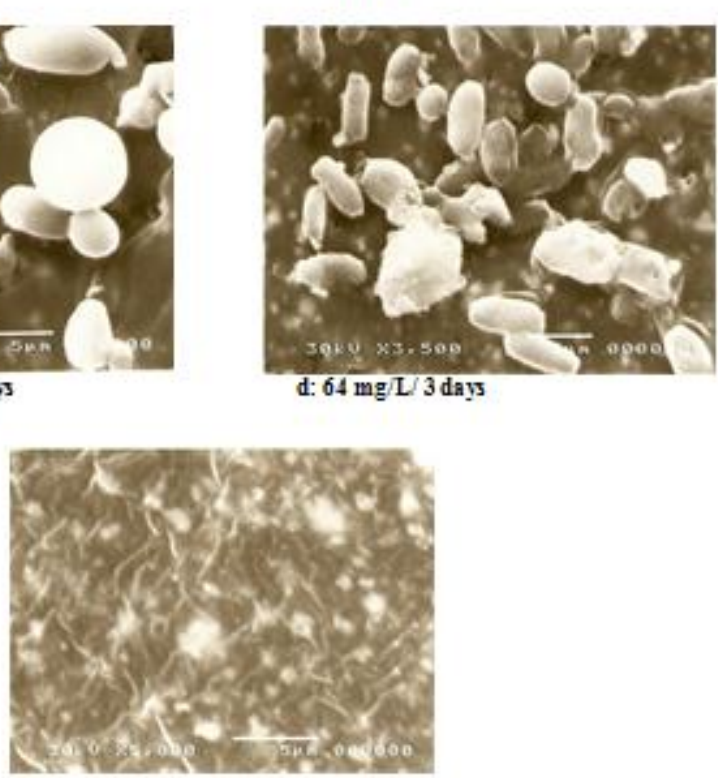

e: $64 \mathrm{mg} / \mathrm{L} / 10$ days

Fig 4. Scanning electron micrographs of $C$. tropicalis biofilm after different lock periods

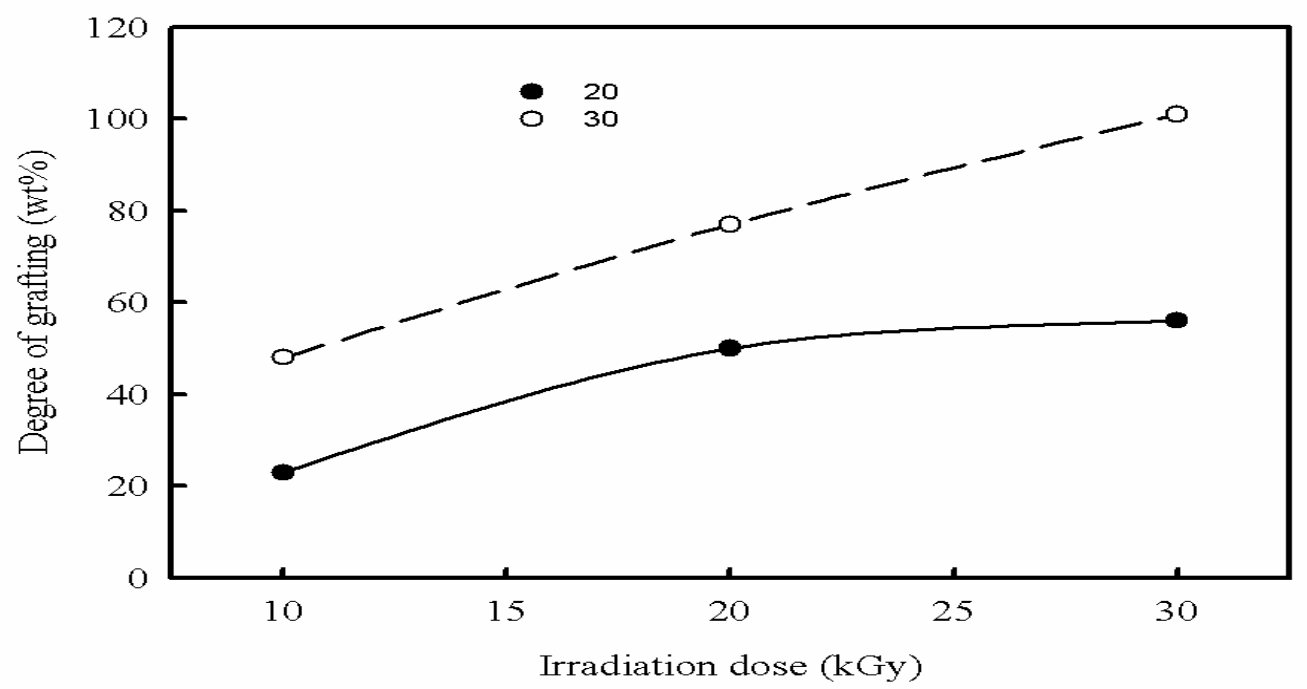

Fig 5. Effect of radiation dose and monomer concentration on grafting yield 


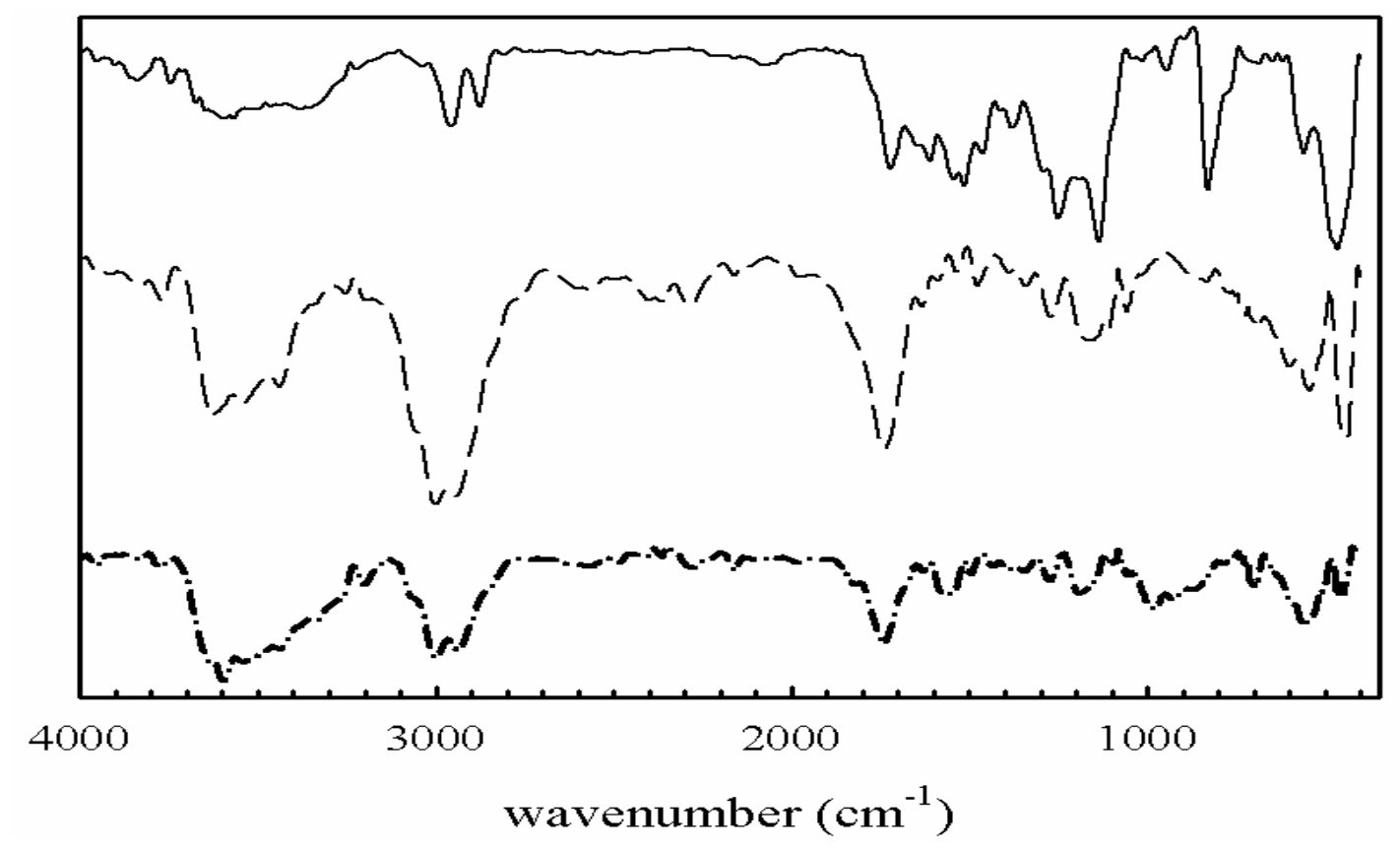

Fig 6. FT-IR spectra of (a) virgin (CVPU), (b) CVPU-g-GMA and (c) gentamicin modified polymer CVPUg-GMA-Gentamicin

P. aeruginosa
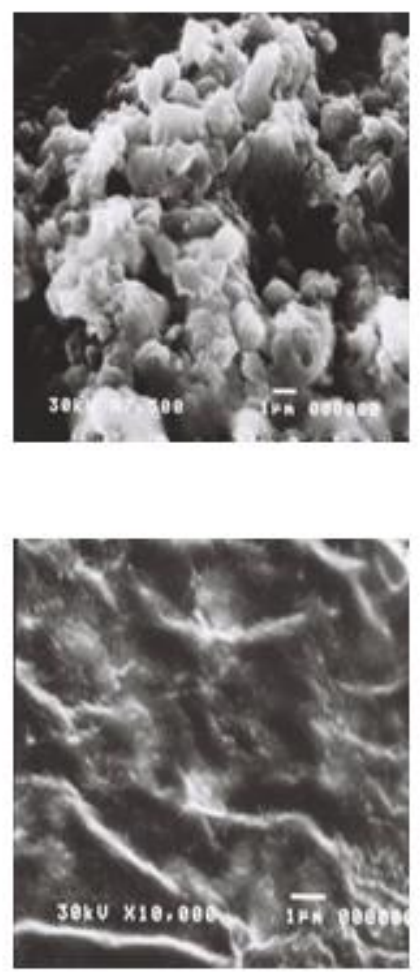

A. baumannü
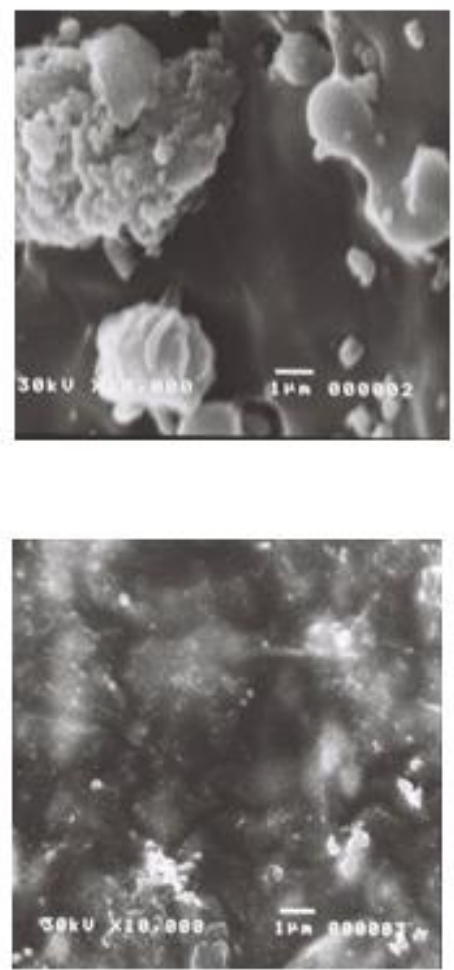

K. pheumonias.
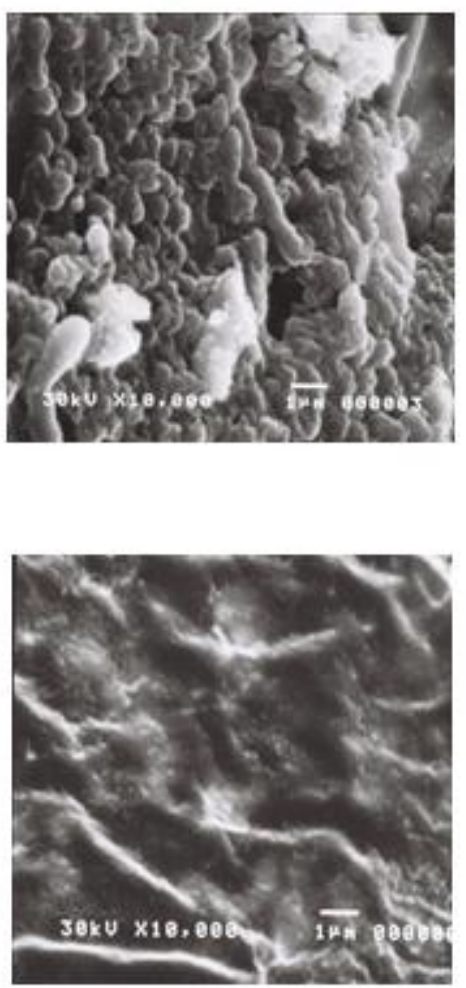

Fig 7. Scanning electron micrographs for commercial CVPU (control) in comparison with gentamicin modified CVPU (CVPU-g-GMA-Gentamicin) after immersion for 30 days in a heavy suspension of test strains 